\title{
Levy Walk Evolution for Global Optimization
}

\author{
O. Urfalıog̃lu \\ Bilkent University \\ Department of Electrical and \\ Electronics Engineering, \\ Ankara/Turkey \\ onay@ee.bilkent.edu.tr
}

\author{
A. E. Çetin \\ Bilkent University \\ Department of Electrical and \\ Electronics Engineering, \\ Ankara/Turkey \\ cetin@bilkent.edu.tr
}

\author{
E. E. Kuruog̃lu \\ ISTI/CNR \\ Signals \& Images Laboratory, \\ Pisa/ltaly \\ kuruoglu@isti.cnr.it
}

\begin{abstract}
A novel evolutionary global optimization approach based on adaptive covariance estimation is proposed. The proposed method samples from a multivariate Levy Skew Alpha-Stable distribution with the estimated covariance matrix to realize a random walk and so to generate new solution candidates in the mutation step. The proposed method is compared to the popular Differential Evolution method, which is one of the best general evolutionary global optimizers available. Experimental results indicate that the proposed approach yields a general improvement in the required number of function evaluations to solve global optimization problems. Especially, as shown in experiments, the underlying heavy tailed alpha-stable distribution enables a considerably more effective global search in more complex problems.

Track: Evolution Strategies
\end{abstract}

\section{Categories and Subject Descriptors \\ G.1.6 [Optimization]: Global Optimization}

\section{General Terms}

Algorithms

\section{Keywords}

Evolutionary Optimization, Global Optimization, AlphaStable Distribution, Heavy Tailed Distribution, Levy Walk

\section{INTRODUCTION}

In many real world optimization problems the cost function is non-seperable and the local optima count is very high. In addition, the local optima are distributed non regularly. In many cases, given the currently discovered local optimum, the next best one is generally not within the close neighbourhood [3].

In this paper, a global optimization method, the Levy Walk

Copyright is held by the author/owner(s).

GECCO'08, July 12-16, 2008, Atlanta, Georgia, USA.

ACM 978-1-60558-130-9/08/07.
Evolution (LWE), is proposed. It is based on multivariate Levy Skew Alpha-Stable distribution (LSASD) with an adaptive covariance matrix and a crossover operator. The LSASD has the interesting property that, depending on the alpha parameter, its variance is infinite. Therefore, a random walk having an alpha-stable distribution involves long range sudden jumps. This property may lead to effective search patterns, especially in complex problems. In the literature, a clonal selection based evolutionary optimization approach using alpha-stable distributions can be found in [5], where it is reported that the proposed approach outperforms traditional Gaussian based mutation operators.

One of the popular evolutionary global optimization methods is Differential Evolution (DE)[4]. It is known for its adaptive mutation scheme, enabling relatively fast global convergence. In this paper, the proposed approach is compared to DE using several well known test problems from the literature.

\section{LEVY WALK EVOLUTION}

Similar to DE, the proposed method called Levy Walk Evolution (LWE) belongs to the class of population based evolutionary global search methods. In contrast to DE, the mutation step is realized by sampling from a Levy Skew Alpha-Stable distribution (LSASD). The LSASD has in general no analytically expressible probability density function. A 1-D LSASD has four parameters $\alpha, \beta, c, \mu$. The $\alpha$ parameter is the exponent and determines the shape of the distribution. For $\alpha=2$ the distribution reduces to a Gaussian distribution, for $\alpha=1$ and $\beta=0$ it reduces to a Cauchy distribution and for $\alpha=0.5$ and $\beta=1$ it reduces to a Levy distribution. The $\beta$ parameter determines the skewness, where $\beta=0$ leads to a symmetric distribution. The parameter $\mu$ determines the mean in the symmetric case. For $0<\alpha<2$, LSASD is a heavy tailed distribution with infinite variance.

In the mutation step of LWE, the weighted difference vector used in DE's mutation step is replaced by a weighted sample from a multivariate LSASD. To enable an efficient global search, scale parameters are adaptively determined by calculating the covariance matrix, denoted by $\Sigma$, from the set of the positions of the particles. This covariance matrix is used in the multivariate LSASD. As a result, the mutation step becomes invariant under coordinate transforms. This invariance can also be found in DE through the difference vector utilization and also in the Evolution Strategy with Covariance Matrix Adaptation (CMA-ES) method [2, 1]. Each component shares the same parameter $\alpha$, which is provided by the user. The $\beta$ parameter and the mean $\mu$ is set 
to zero, respectively, so that only symmetric distributions with zero mean are utilized.

In LWE, new positions for the iteration step $i+1$ are determined by

$$
\begin{aligned}
\boldsymbol{y}_{n, i+1} & =\boldsymbol{x}_{k, i}+F \cdot \boldsymbol{s}_{n, i} \\
\boldsymbol{x}_{n, i+1} & =C\left(\boldsymbol{x}_{n, i}, \boldsymbol{y}_{n, i+1}\right),
\end{aligned}
$$

where $k$ is a random integer from the discrete set $\{1, \ldots, S\}$ and $F$ is a weighting scalar. The additive vector $\boldsymbol{s}_{n, i}$ is sampled from a LSASD. The vectors $\boldsymbol{y}_{n, i+1}$ and $\boldsymbol{x}_{n, i}$ are used in the crossover operation $C\left(\right.$ ), which yields $\boldsymbol{x}_{n, i+1}$. In the following, the method of generating samples from a multivariate LSASD is described.

1. Calculate covariance matrix $\Sigma$ from the positions of particles.

2. Calculate the Cholesky decomposition of $\Sigma: \Sigma=L L^{\top}$.

3. For a D-dimensional multivariate LSASD, sample $\tau_{j}, j=1, \ldots, D$ each from a 1 -D LSASD having the parameters $c=1, \mu=\beta=0$ and the specified value for the parameter $\alpha$.

4. Calculate multivariate sample $s=L \cdot\left(\tau_{1}, \ldots, \tau_{D}\right)^{\top}$.

Steps 1 and 2 are only executed when there is progress, i.e. one or more particles achieve new positions having lower costs. This mutation scheme incorporates a Gaussian random walk for $\alpha=2$ and a Cauchy random walk for $\alpha=1$, respectively. For simplicity, in this paper the general case with an arbitrary $\alpha \in] 0,2]$ is called Levy walk.

\section{EXPERIMENTS}

In the test suite, unimodal as well as seperable and nonseperable multimodal cost functions $f_{i}(x)$ with regularly and non-regularly distributed local optima are utilized. For each cost function $f_{i}(x)$, the function value $y$ at the global optimum $\hat{x}$ is managed to be zero

$$
f_{i}(\hat{\boldsymbol{x}})=0 .
$$

The global convergence criterium is $f_{i}(\tilde{\boldsymbol{x}}) \leq 0.001$. There are three parameters for the DE method to be selected to find an optimal setting. These are the population size $S$, the differential vector weight $F$ and the crossover probability $p$. On the other hand, the proposed LWE method has four parameters to be adjusted. These include the population size $S$, the step size weight $F$, the parameter $\alpha$ and the crossover probability $p$. For each cost function, the free parameters are adjusted such that the required number of mean function evaluations (MFE) is minimized and no global convergence failure occurs within 200 repetitions of the global search. Figure. 1 shows experimentally determined results for the MFE's.

\section{CONCLUSIONS}

Compared to the DE-method, the proposed Levy Walk Evolution (LWE) shows superior performance regarding the number of mean function evaluations in unimodal and complex non-seperable problems, where the local optima are distributed non-regularly. It is experimentally observed that multivariate heavy tailed distribution based mutation with adaptive covariance matrix turns out to have the capability of escaping local optima even with a considerably less
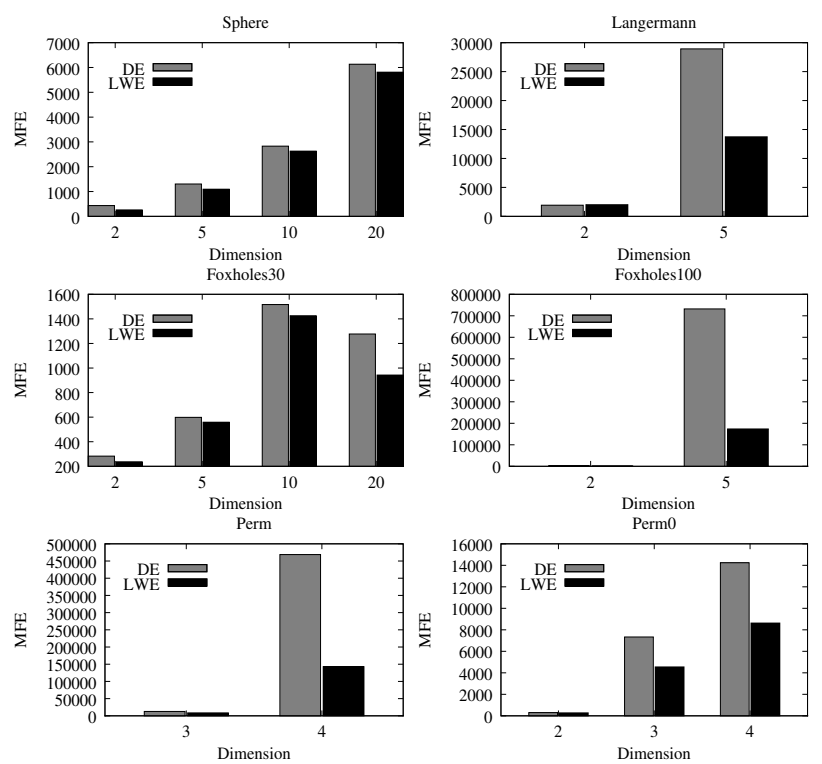

Figure 1: Required MFE for several cost functions: DE vs. $L W E$

amount of particles. This is one of the reasons for the good performance of LWE on such complex problems.

As a general rule, decreasing the $\alpha$ parameter in LWE corresponds to heavier tailed distributions and this naturally enhances global search.

Another possible use case for alpha-stable distribution based evolutionary optimization methods could be found in robust optimization.

\section{ACKNOWLEDGEMENTS}

This work is supported in part by TÜBITAK under the grant numbers EEEAG-105E065, 105E121 and AT FP6507752 (MUSCLE-NoE).

\section{REFERENCES}

[1] N. Hansen, S. D. Müller, and P. Koumoutsakos. Reducing the time complexity of the derandomized evolution strategy with covariance matrix adaptation (cma-es). Evol. Comput., 11(1):1-18, 2003.

[2] N. Hansen and A. Ostermeier. Adapting arbitrary normal mutation distributions in evolution strategies: the covariance matrix adaptation. In Proc. of the 1996 IEEE Int. Conf. on Evolutionary Computation, pages 312-317, Piscataway, NJ, 1996. IEEE Service Center.

[3] A. Lagnoux. Rare event simulation. Probability in the Engineering and Informational Sciences, 20(1):43-66, 2006.

[4] K. V. Price. Differential evolution: a fast and simple numerical optimizer. In Biennial Conference of the North American Fuzzy Information Processing Society, NAFIPS, pages 524-527. IEEE Press, New York. ISBN: 0-7803-3225-3, June 1996.

[5] K. Trojanowski. Clonal selection approach with mutations based on symmetric alpha-stable distributions for non-stationary optimization tasks. Adaptive and Natural Computing Algorithms, 4431/2007:184-193, july 2007. 